\title{
Zunyou Wu, China CDC's Chief Expert of Epidemiology
}

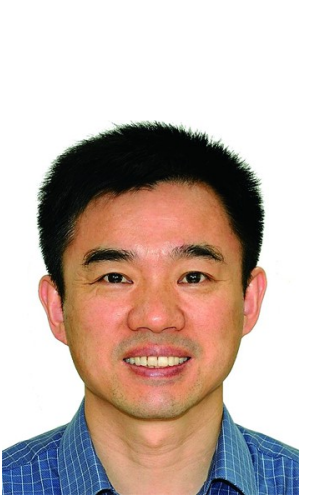

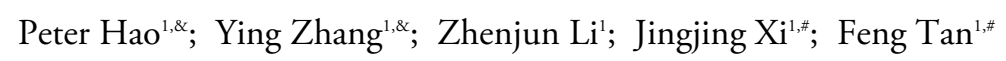

implementation and expansion of a comprehensive HIV response in China and the development of internationally collaborative efforts to bring the global HIV epidemic under control.

From the very beginning, Zunyou Wu has been a leader in China's HIV response. After completing his Ph.D. in the United States at UCLA in 1995, Zunyou Wu returned to China and quickly became involved in a major HIV outbreak investigation in rural Anhui province. The investigation revealed the greatest public health tragedy China has faced in a century - the widespread HIV infection of paid plasma donors in villages throughout central China. Zunyou Wu was the epidemiologist on the team that identified unsafe plasma collection procedures as the common origin of a large number of newly-identified HIV cases. This timely discovery spurred immediate actions to end unsafe plasma collection methods and prevented thousands of donors from acquiring HIV infections.

Immediately following the conclusion of the outbreak investigation, Zunyou Wu started to promote HIV testing as a control strategy. He conducted the first HIV survey among former commercial plasma donors in 1996, which revealed that HIV prevalence in this population was $12.5 \%$. He then pushed for the rapid expansion of treatment services for those who had become infected with HIV through plasma donation and the implementation of premarital health screening to prevent the spread of infection. From 1997 to 1999, Zunyou Wu spearheaded China's first large-scale HIV training program for healthcare workers in rural areas, and from 2003 to 2007, he led a US National Institutes of Health (NIH)-funded, community-based, intervention trial to reduce HIV/AIDS-related stigma for infected former plasma donors.

To realize these successes, Zunyou Wu worked vigorously within China CDC and across sectors to push for changes in the national HIV response that required a significant shift in long-standing social and political paradigms. For example, China's HIV epidemic originated and grew rapidly early on among people who use drugs (PWUD) in southwestern China as a result of unsafe injecting behavior. Using implementation science strategies to scale-up harm reduction programs for reducing HIV transmission among people who inject drugs (PWID) in China, Zunyou Wu led the design, pilot testing, and scale-up of methadone maintenance treatment (MMT) and needle exchange programs in China. These harm reduction programs have become national strategies and resulted in the continuing decline of HIV incidence and prevalence among PWUD in China. The harm reduction programs in China led by Zunyou Wu have become internationally-recognized best practices for controlling HIV among PWUD. Zunyou Wu has become a preeminent expert on the control of HIV epidemics among drug users. He has shared lessons learned and best practices with delegates from Russia, the Ukraine, Myanmar, Vietnam, and Thailand, thereby contributing directly to the testing, treatment, and prevention of HIV among PWUD globally.

Zunyou Wu used implementation science strategies to pilot and scale-up a simplified protocol for HIV testing and treatment initiation. Although HIV testing has been expanded and more people living with HIV (PLWH) are being diagnosed, a considerably high proportion of HIV infections are diagnosed at late clinical stages and patients were dying shortly after diagnosis. The complexity of multiple stages and multiple institutes involved in HIV diagnosis and treatment had made the process from initially screening HIV reactivity to finally starting antiretroviral therapy (ART) difficult and slow, resulting in unacceptably high rates of loss to follow-up in the pre-ART period. 
He pilot tested a structural intervention called the "One4All" strategy, which simplified HIV diagnosis and treatment initiation. He first used a pre/post study design, and then conducted a cluster-randomized trial, supported by the US National Institute on Drug Abuse (NIDA) of the NIH. Both approaches demonstrated that the simplified protocol significantly shortened the time interval between screening for HIV-reactivity and initiating ART and significantly reduced mortality among newly-diagnosed HIV cases. The "One4All" strategy has since been adopted as China's national strategy and written into the $13^{\text {th }}$ Five-Year Action Plan as a key control strategy for China's national HIV/AIDS response.

Zunyou Wu continued his promotion of HIV testing as an important HIV/AIDS control strategy starting from the mid-2000s. More recently, it had become apparent that a very high proportion of HIV-infected individuals who remained undiagnosed, which meant they not only failed to receive the medical care they needed but were also contributing to ongoing transmission. Zunyou Wu led several studies demonstrating the importance of promoting HIV testing. He has also provided strong evidence for the importance of improving retention in the care continuum from diagnosis to treatment to viral suppression both for individuals' clinical benefit and for communities' public health benefit. Zunyou Wu's strong and steadfast advocacy for expanded HIV testing coverage and strategies has meant that HIV testing remains a key control strategy in China's national HIV/AIDS response programs still today.

Zunyou $\mathrm{Wu}$ created the National HIV/AIDS Comprehensive Response Information Management System (CRIMS) in 2008. CRIMS is a nationwide online electronic medical record system that integrates data on HIV case reporting, surveillance, testing, prevention, ART, and MMT. CRIMS is a key tool for monitoring HIV/AIDS strategy implementation in China.

Participating in the response to the SARS outbreak in Beijing in 2003, Zunyou Wu discovered long time delays from onset of illness to patient isolation and proposed to shorten the time between the first clinic visit and hospitalization as an important containment strategy. His proposed changes to case management protocols were immediately adopted, helping achieve control of SARS in Beijing. Zunyou Wu attended the $56^{\text {th }}$ World Health Assembly and contributed to drafting the international resolution on SARS (WHA56.28 Revision of the International Health Regulations; WHA56.29 Severe acute respiratory syndrome, SARS).

Zunyou Wu has participated in China's response to COVID-19 since January 16, 2020. He analyzed epidemic data in real time as it was collected in the early days of the outbreak in Wuhan and has since monitored epidemic trends closely. He has made significant contributions to the global understanding of COVID-19 epidemiology, particularly the heightened vulnerability of the elderly and people with underlying chronic conditions, and has also studied and reported on the important role of asymptomatic and pre-symptomatic infection in transmission. Zunyou Wu was involved in the World Health Organization (WHO) - China Joint Mission on COVID-19 from February 16-24, 2020. He worked with WHO technical experts and leaders, participated in field visits, and contributed to drafting the final Joint Mission report. Additionally, Zunyou Wu was involved in investigating the COVID-19 outbreak in Beijing in June and July 2020 and found that the cause of the outbreak may have been associated with contamination of imported seafood products.

As China CDC's Chief Expert of Epidemiology, Zunyou Wu continues to lead the country's response to infectious diseases by providing critical insight and experience. His experiences addressing HIV/AIDS, SARS, and COVID-19 demonstrate his invaluable contributions to academic research and to informing the public.

doi: $10.46234 / \mathrm{ccdcw} 2020.175$

\# Corresponding authors: Jingjing Xi, xijj@chinacdc.cn; Feng Tan, tanfeng@chinacdc.cn.

\footnotetext{
${ }^{1}$ Chinese Center for Disease Control and Prevention, Beijing, China.

\& Joint first authors.
}

Submitted: August 07, 2020; Accepted: August 14, 2020 